\title{
Chromospheric Activity in 55 Cancri: I. Results from Theoretical Wave Studies
}

\author{
Diaa E. Fawzy ${ }^{1}$ and Manfred Cuntz ${ }^{2 \star}$ \\ ${ }^{1}$ Faculty of Engineering, Izmir University of Economics, 35330 Izmir, Turkey \\ ${ }^{2}$ Department of Physics, University of Texas at Arlington, Arlington, TX 76019, USA
}

Accepted XXX. Received YYY; in original form ZZZ

\begin{abstract}
We present theoretical models of chromospheric heating for 55 Cancri, an orange dwarf of relatively low activity. Self-consistent, nonlinear and time-dependent ab-initio numerical computations are pursued encompassing the generation, propagation, and dissipation of waves. We consider longitudinal waves operating among arrays of flux tubes as well as acoustic waves pertaining to nonmagnetic stellar regions. Additionally, flux enhancements for the longitudinal waves are also taken into account as supplied by transverse tube waves. The Ca II K fluxes are computed (multi-ray treatment) assuming partial redistribution as well as time-dependent ionization. The self-consistent treatment of time-dependent ionization (especially for hydrogen) greatly impacts the atmospheric temperatures and electron densities (especially behind the shocks); it also affects the emergent Ca II fluxes. Particularly, we focus on the influence of magnetic heating on the stellar atmospheric structure and the emergent Ca II emission, as well as the impact of nonlinearities. Our study shows that a higher photospheric magnetic filling factor entails a larger Ca II emission; however, an increased initial wave energy flux (e.g., associated with mode coupling) is of little difference. Comparisons of our theoretical results with observations will be conveyed in forthcoming Paper II.
\end{abstract}

Key words: methods: numerical - stars: chromospheres - stars: magnetic fields - stars: individual (55 Cnc) - magnetohydrodynamics (MHD)

\section{INTRODUCTION}

In the previous decades a large number of studies has been presented indicating the significance of wave heating in outer stellar atmospheres for stars of different activity levels, including relatively inactive stars (e.g., Narain \& Ulmschneider 1996, including references therein and subsequent work); see also more recent observations by Freij et al. (2014) and Kayshap et al. (2018). The general picture that emerged indicates that the atmospheres of high-activity stars are dominantly heated by magnetic processes (including magnetic waves), whereas for the atmospheric heating of low-activity stars nonmagnetic processes may play a more prominent role (e.g., Schrijver 1995; Buchholz et al. 1998; Rammacher \& Cuntz 2003), although the role of magnetic phenomena in those stars is expected to be significant as well (e.g., Judge \& Cuntz 1993; Judge \& Carpenter 1998). When stars age, the relative importance of atmospheric magnetic processes tends to subside, a process closely related to the evolution of angular momentum (e.g., Marilli et al. 1986; Hartmann \& Noyes 1987; Keppens et al. 1995; Charbonneau et al. 1997; Wolff \& Simon 1997; Mittag et al. 2018). Consequently, main-sequence stars of advanced age, as e.g. old $\mathrm{G}$ and $\mathrm{K}$ stars, are targets of great interest for the study of the relative importance of magnetic and non-magnetic wave atmospheric heating.

As pointed out by, e.g., Linsky (1983), Schrijver \& Zwaan (2000), as well as a large array of other work, magnetic heating in stellar

\footnotetext{
^ E-mail: cuntz@uta.edu (MC)
}

atmospheres is strongly correlated to increased outer atmospheric emission. Increased stellar rotation usually entails an increased photospheric magnetic flux $\left(\propto B_{0} f_{0}\right)$, where $B_{0}$ is the photospheric magnetic field strength and $f_{0}$ is the magnetic filling factor. Therefore, in a statistical sense, it is possible to link $B_{0} f_{0}$ to both the stellar rotation period $P_{\text {rot }}$ and age (e.g., Noyes et al. 1984; Marcy \& Basri 1989; Montesinos \& Jordan 1993; Saar 1996), on the one hand, and to the emergent chromospheric emission flux (e.g., Schrijver et al. 1989; Jordan 1997; Fawzy et al. 2002b), on the other hand.

In this work, we pursue studies for 55 Cancri $\left(55 \mathrm{Cnc}, \rho^{1} \mathrm{Cnc}\right.$ ), a G8 V star (Gonzalez 1998). $55 \mathrm{Cnc}$ is an old main-sequence star with a mass and luminosity considerably smaller than the Sun and a slow rotation rate (see below); this star also meets the characteristics of an orange dwarf. In this work, we study the relative importance of magnetic heating and non-magnetic heating (i.e., acoustic waves) in consideration of 55 Cnc's low activity level, including the emergence of chromospheric emission. Our paper is structured as follows: In Section 2, we convey our theoretical approach, including a description of the acoustic and magnetic wave energy generation, the flux tube models, and the computation of the emergent Ca II emission fluxes in response to our wave calculations. In Section 3, we present our results and discussion, including relevant aspects of time-averaged atmospheres. In Section 4, we give our summary and conclusions. 
Table 1. Stellar Parameters

\begin{tabular}{lcl}
\hline Parameter & Value & Reference \\
\hline Spectral Type & G8 V & Gonzalez (1998) \\
Effective Temperature (K) & 5165 & Ligi et al. (2016) \\
$\ldots$ & 5172 & Yee et al. (2017) \\
Color $(B-V)$ & 0.87 & Hoffleit \& Warren (1995) \\
Mass $\left(M_{\odot}\right)$ & 0.91 & von Braun et al. (2011) \\
Surface Gravity (log cgs) & 4.37 & Gray et al. (2003) \\
$\ldots$ & 4.43 & Yee et al. (2017) \\
Metallicity [Fe/H] & 0.35 & Yee et al. (2017) \\
Age (Gyr) & 8.6 & Mamajek \& Hillenbrand (2008) \\
$\ldots$ & 10.2 & von Braun et al. (2011) \\
Rotation Period (d) & 42.2 & Henry et al. (2000) \\
$\ldots$ & 38.8 & Bourrier et al. (2018) \\
\hline
\end{tabular}

Note: See references for information on the uncertainty bars and background information on the adopted methodology. No claim is made about the completeness of this list.

Table 2. Summary of Acronyms

\begin{tabular}{ll}
\hline Acronym & Definition \\
\hline ACW & Acoustic Wave \\
LTE & Local Thermodynamic Equilibrium \\
LTW & Longitudinal Flux Tube Wave \\
MFF & Magnetic Filling Factor $\left(f_{0}\right)$ \\
NLTE & Non-Local Thermodynamic Equilibrium \\
NTDI & Non-Time-Dependent Ionization \\
PRD & Partial Redistribution \\
TDI & Time-Dependent Ionization \\
TTW & Torsional Flux Tube Wave \\
\hline
\end{tabular}

\section{THEORETICAL APPROACH}

\subsection{Stellar Parameters}

In this work, we pursue theoretical atmospheric studies of $55 \mathrm{Cnc}$, a G8 V star (Gonzalez 1998); see Table 1 for details. A recent study by von Braun et al. (2011) conveys a mass of $0.91 M_{\odot}$; hence, 55 Cnc can be classified as an orange dwarf; those stars characterized by a mass range of about $0.80 \pm 0.13$ (Gray 2005). Furthermore, $55 \mathrm{Cnc}$ is considerably older than the Sun. Previous estimates of 55 Cnc's age are 8.6 Gyr (Mamajek \& Hillenbrand 2008) and 10.2 Gyr (von Braun et al. 2011); see Bourrier et al. (2018) for discussion.

Previous work about the stellar rotation period indicates values of $42.2 \mathrm{~d}$ (Henry et al. 2000) and $38.8 \mathrm{~d}$ (Bourrier et al. 2018); clearly, these values of slow rotation are closely connected to 55 Cnc's advanced age, see, e.g., Skumanich (1972) and subsequent work. In the framework of our models, the stellar rotation period as previously derived also allows default estimates of the stellar photospheric magnetic filling factor (see Sect. 2.3).

\subsection{Acoustic and Magnetic Wave Energy Generation}

A crucial aspect of this study pertains to the calculation of the initial acoustic and magnetic wave energy fluxes; notably, model simulations for the generation of longitudinal flux tube waves
$(\mathrm{LTWs})^{1}$. Regarding acoustic waves (ACWs), previous calculations for wave energy fluxes have been given by Musielak et al. (1994) and Ulmschneider et al. (1996). The latter authors computed both acoustic frequency spectra and total acoustic fluxes on the basis of mixing-length convection zone models for a large range of stars, including main-sequence stars. The results were identified to depend on the stellar effective temperature, surface gravity, metallicity and the mixing-length parameter $\alpha_{\mathrm{ML}}$.

Following subsequent work by Stein et al. (2009a,b), who employed detailed 3-D convective models, the value of $\alpha_{\mathrm{ML}}=1.8$ has become increasingly established; it has also been previously used by Fawzy et al. (2012). For $55 \mathrm{Cnc}$ this approach yields an acoustic wave energy flux of $F_{\mathrm{AC}}=3.3 \times 10^{7} \mathrm{erg} \mathrm{cm}^{-2} \mathrm{~s}^{-1}$ with a representative wave period of $P_{\mathrm{AC}}=60 \mathrm{~s}$. This latter value has also been adopted for our monochromatic wave simulations intended for comparison with the more detailed models based on wave spectra; see Fig. 1 for results on the acoustic wave energy spectra.

Akin to our previous work, we calculate wave energy fluxes and spectra for LTWs based on previous work by Musielak et al. (1995), Ulmschneider \& Musielak (1998), and Ulmschneider et al. (2001), among others. In this approach, stellar turbulence is described via an extended Kolmogorov turbulent energy spectrum and the modified Gaussian frequency factor; see Musielak et al. (1995) for a detailed discussion of the potential and limitations of this approach. According to that work, the interaction between the magnetic flux tubes and the surrounding turbulent medium results in the generation of LTWs, among other types of waves.

At the start of the procedure, initial stellar magnetic flux tube models are constructed (see Sect. 2.3) with the external pressure fluctuations considered to be responsible for the squeezing of the flux tube; they are represented by a superposition of a sufficiently high number of partial waves. Again, an extended Kolmogorov spatial turbulent energy spectrum and the modified Gaussian frequency factor is applied. Using the stellar parameters of $55 \mathrm{Cnc}$ as input (see Sect. 2.1), we find $F_{\mathrm{LTW}}=1.7 \times 10^{8} \mathrm{erg} \mathrm{cm}^{-2} \mathrm{~s}^{-1}$ (upward directed flux) for a magnetic field strength of $B_{0}=1698 \mathrm{G}$ (see Sect. 2.3 ), independent of the value of the filling factor $f_{0}$, with a representative wave period of $P_{\mathrm{LTW}}=60 \mathrm{~s}$ as also obtained for acoustic waves. The wave frequency spectrum for LTWs is given in Fig. 1.

\subsection{Flux Tube Models}

The nonlinear interactions between the magnetic structures given as flux tubes on the stellar surface and the surrounding turbulence entail the generation of waves that are able to heat the stellar atmospheres, see, e.g., Ulmschneider et al. (2001) and related studies. This approach has also been implemented for the current computations. The computations are started by constructing thin magnetic flux tubes that are vertically oriented in a magnetic-free surrounding. Therefore, we need to specify four basic parameters; namely, the stellar effective temperature $T_{\text {eff }}$, the surface gravity $g$, the magnetic field strength at the stellar surface $\mathrm{B}_{0}(z=0 \mathrm{~km})$, and the surface magnetic filling factor $f_{0}(z=0 \mathrm{~km})$. Regarding our models, the latter determines the geometry of the magnetic flux tubes as well as the tube spreading at the upper layers. It is noteworthy that the efficiency of wave heating of the upper layers critically depends on the flux tube geometry (e.g., Fawzy et al. 1998).

The value of the magnetic field strength, $\mathrm{B}_{0}(z=0 \mathrm{~km})$, is of critical importance for the generation of the magnetic wave energy

\footnotetext{
1 See Table 2 for a summary of the acronyms.
} 

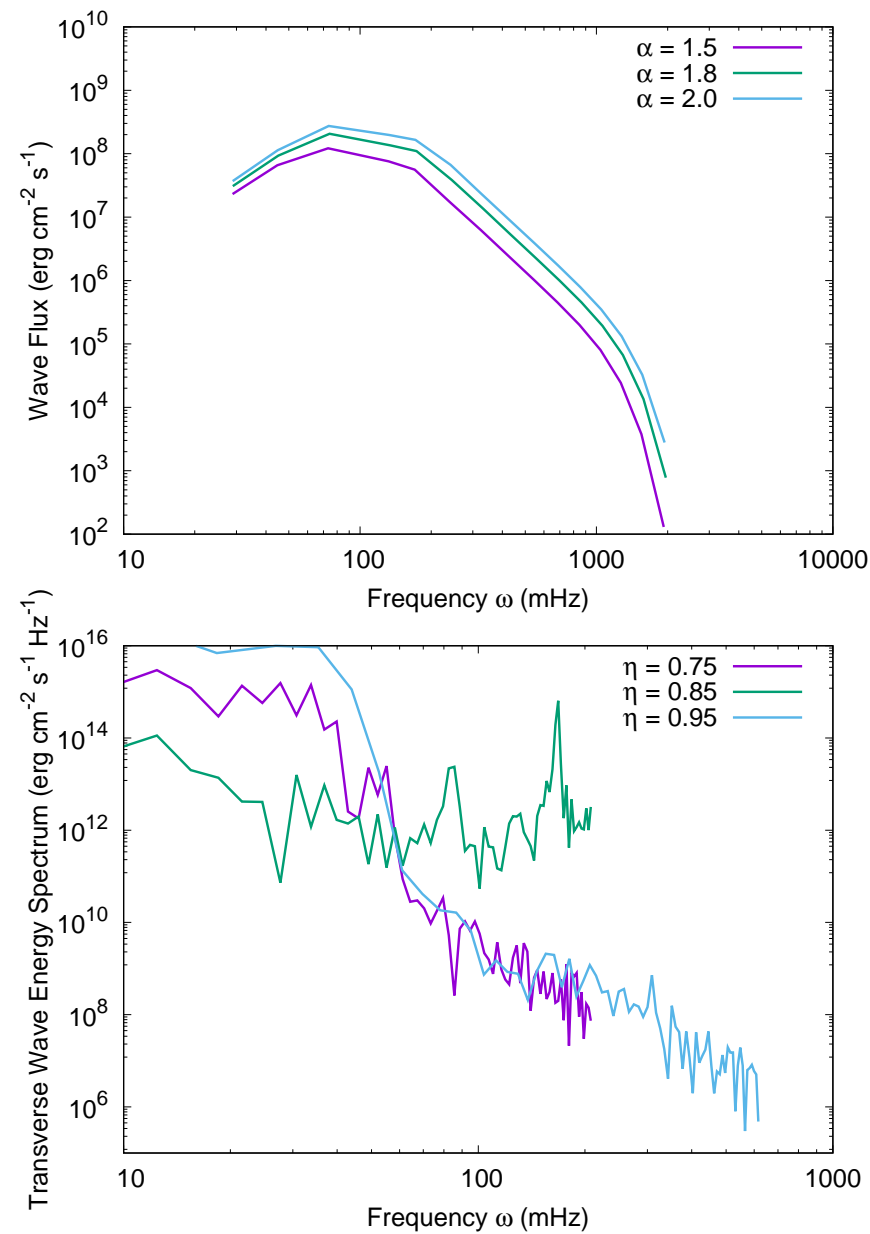

Figure 1. Initial wave energy spectra for acoustic wave models (top) and longitudinal flux tube models (bottom).

as the efficiency decreases for rigid tubes of higher field strengths. The determination of the value $B_{0}(z=0 \mathrm{~km})$ from observations is not straightforward; however, the value of $B_{0} f_{0}$ can be determined with a relatively high accuracy ${ }^{2}$. For the current computations, we use the solar case as guidance, where the magnetic field strength at the base of the flux tube is readily assumed as $\eta=B_{0}(z=0 \mathrm{~km}) / B_{\text {eq }}$ $=0.85$ of the equipartition field $\left(B_{\text {eq }}\right)$; see, e.g., Fawzy \& Cuntz (2011) for an analysis on the impact of $\eta$ on LTW simulations for different types of stars.

The equipartition field is the maximum allowed magnetic field strength for quasi-vacant tubes; it is computed from the horizontal pressure balance equation given by

$\frac{B^{2}(z)}{8 \pi}+P_{i}(z)=P_{e}(z)$

(e.g., Spruit 1981; Herbold et al. 1985). In order to compute the equipartition field strength $B_{\text {eq }}$, we set the internal gas pressure $P_{i}(z=0 \mathrm{~km})=0$, with the external gas pressure determined from our initial models; here $P_{e}(z=0 \mathrm{~km})=1.59 \times 10^{5}$ dyne $\mathrm{cm}^{-2}$ entails $B_{\text {eq }}(z=0 \mathrm{~km})=1998 \mathrm{G}$. The magnetic filling factor

\footnotetext{
2 Main-sequence stars of different activity levels but of the same spectral type are expected to exhibit (approximately) the same value of $B_{0}$, as set by the stellar photospheres. Yet increased activity levels typically correspond to larger photospheric magnetic filling factors $f_{0}$; e.g., Johns-Krull \& Valenti (1996, 2000), See et al. (2019), among other literature.
}

$f_{0}(z=0 \mathrm{~km})$ can be computed from the empirically determined relationship between $B_{0} f_{0}$ and the rotational period $P_{\text {rot }}$ given as

$B_{0} f_{0}(z=0 \mathrm{~km})=238-5.51 P_{\text {rot }}$.

The latter relation has been deduced by Cuntz et al. (1999) based on data from Rüedi et al. (1997). Taking $B_{0}=0.85 B_{\text {eq }}$ enables us to compute the value of the magnetic filling factor at the stellar surface. For the current computations, we consider two values of $P_{\text {rot }}=42.2$ days and 38.8 days; see Henry et al. (2000) and Bourrier et al. (2018), respectively. The corresponding photospheric magnetic filling factors are given as $f_{0}=0.3 \%$ and $1.4 \%$, respectively. Figure 2 shows the two constructed magnetic flux tubes based on these magnetic filling factors.

\subsection{Main Radiative Contributors}

The main radiative contributors included in the current computations stem from the $\mathrm{H}^{-}, \mathrm{Ca}$ II, and $\mathrm{Mg}$ II ions. The radiative losses from singly ionized two-level atoms, i.e., the Ca II $\mathrm{K}$ and $\mathrm{Mg}$ II $k$ lines, are scaled to represent the respective multi-level atoms.

The underlying atomic models entail the five-level-based $\mathrm{Ca}$ II lines (K, H, IRT) and the three-level-based Mg II lines ( $k$ and $h$ ), besides the respective continua; the scaling is done in order to accurately account for the total radiation losses. The following factors have been adopted: for $\mathrm{Mg}$ II, the factors of 1.50 and 1.53 as used for monochromatic waves and spectral waves, respectively, and for Ca II, 


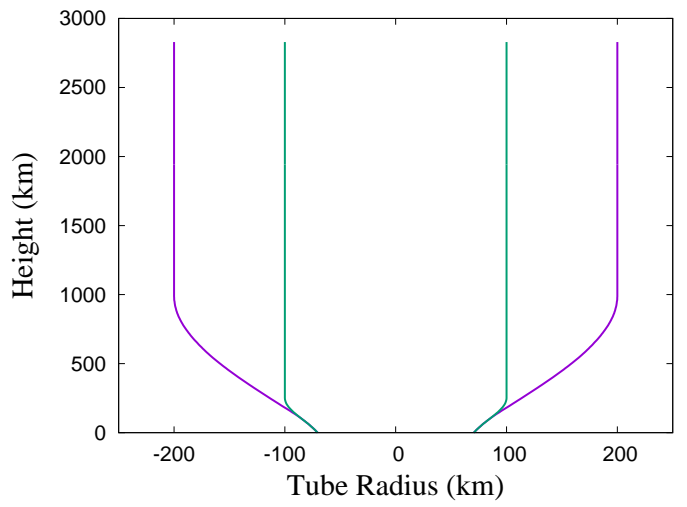

Figure 2. Initial magnetic flux tube models for $55 \mathrm{Cnc}$. The photospheric magnetic filling factor (main models) is given as $f_{0}=0.3 \%$ (purple). We also discuss some models with $f_{0}=1.4 \%$ for comparison (green). The tube opening radii are given as $100 \mathrm{~km}$ and $200 \mathrm{~km}$, respectively.

the factors of 4.71 and 4.68. The total radiative damping function (in

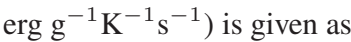

$$
\left.\frac{d S}{d t}\right|_{\mathrm{Rad}}=\left.\frac{d S}{d t}\right|_{\mathrm{H}^{-}}+\left.\frac{d S}{d t}\right|_{\mathrm{Ca} \text { II }}+\left.\frac{d S}{d t}\right|_{\mathrm{Mg} \mathrm{II}} .
$$

Previously, state-of-the-art models, see Rammacher \& Ulmschneider (2003) for the Sun and Fawzy (2015) for other late-type stars, have been developed to derive sets of radiation correction factors. These allow the consideration of adequate radiative energy losses given by multi-level atomic models in the context of time-dependent wave computations. Figure 3 shows the radiation damping functions for $\mathrm{Ca}$ II $\mathrm{K}$ and $\mathrm{Mg}$ II $k$ lines as a function of height and for an LTW model with a magnetic filling factor of $f_{0}=1.4 \%$. Generally, it is found that the formation heights for Ca II and Mg II range between for $700 \mathrm{~km}$ and 1800 $\mathrm{km}$, somewhat depending on the model. Radiative energy losses are most pronounced behind strong shocks owing to the impact of shock-shock interaction (see Sect. 3.1) and in models with time-dependent hydrogen ionization omitted (see Sect. 3.2).

In the following, we will mostly study $\mathrm{Ca}$ II, even though radiative energy losses by $\mathrm{Mg}$ II are relevant as well; see Paper II for a more extended analysis. For example, for the LTW-NTDI model with $f=1.4 \%$ at an elapsed time of $3914 \mathrm{~s}$, we find for the ratio (absolute values) of the radiative damping function $\mathrm{Mg}$ II $k$ / Ca II K values between $5.4 \times 10^{-3}$ (minimum) and $2.1 \times 10^{2}$ (maximum), with an average given as 1.13 , depending on the atmospheric height.

\subsection{Computation of the Emergent Ca II Emission Fluxes}

A pivotal aspect of time-dependent wave simulations, both pertaining to LTWs and ACWs, is the calculation of the chromospheric emission based on a detailed implementation for Ca II. The chromospheric Ca II fluxes are computed by considering non-LTE (NLTE) and partial redistribution (PRD) radiative transfer. For the adequate representation of the multi-level atomic model of $\mathrm{Ca}$ II, we rely on previous results by Fawzy (2015).

In this work, the radiation losses by two-level atoms of Ca II K line has been scaled up through the usage of correction factors depending on the type of model, which have been calculated and tested based on detailed radiative transfer models. The current computations are based on multi-ray 1.5-D radiative transfer assuming PRD. The flux tubes are assumed to be distributed uniformly in a network structure
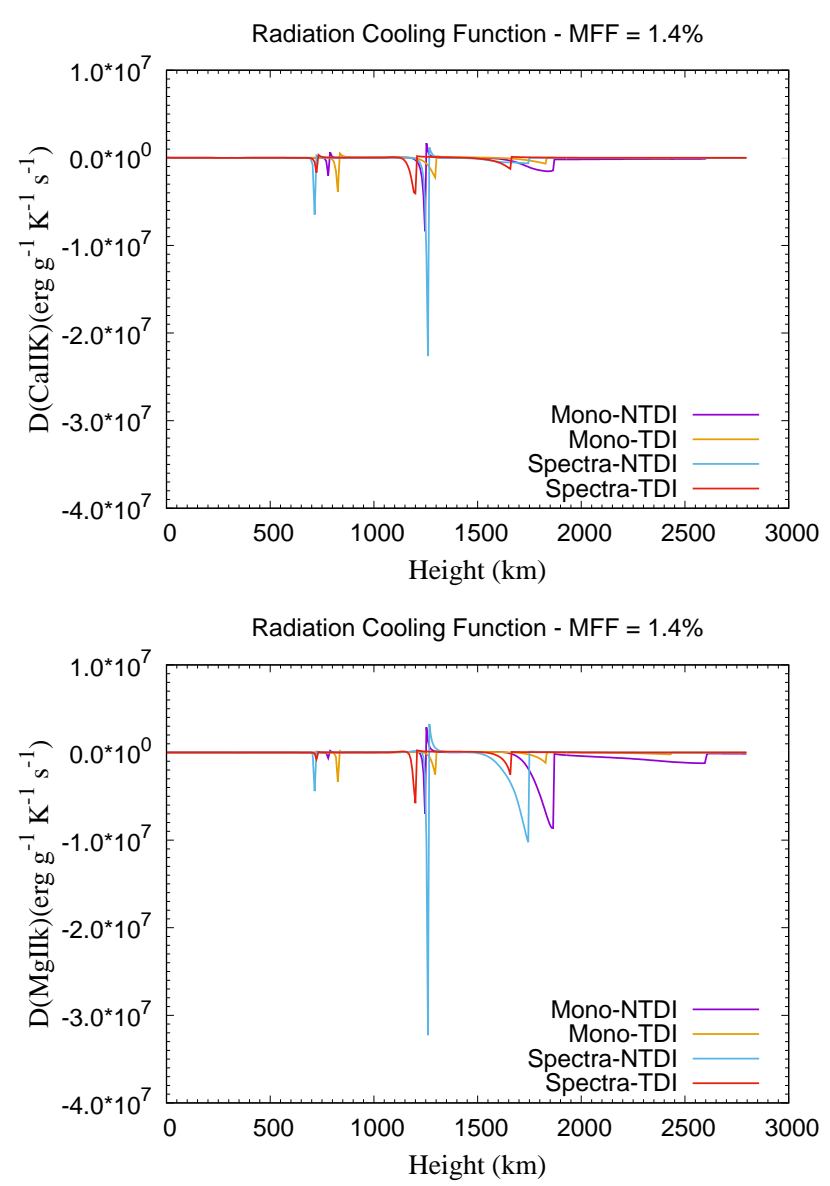

Figure 3. Radiation damping functions regarding the Ca II $\mathrm{K}$ and $\mathrm{Mg}$ II $k$ lines for different models with $f_{0}=1.4 \%$ and an elapsed time of $3940 \mathrm{~s}$.

over the star's surface. The flux tubes are assumed to be heated by LTWs and the voids (i.e., non-magnetic regions) by ACWs. To ensure that the line fluxes represent the different wave phases, the atmospheres inside the flux tubes are filled randomly with different wave phases out of total 10 phases. The ACW models are intended to describe basal chromospheric heating.

To ensure that all surface areas contribute to the computations of the disk-integrated $\mathrm{Ca}$ II $\mathrm{K}$ emission fluxes, we consider five angles, which are: $\theta=26^{\circ}, 46^{\circ}, 60^{\circ}, 73^{\circ}$, and $84^{\circ}$. This approach assures that all surface regions appropriately contribute to the overall Ca II fluxes.

\section{RESULTS AND DISCUSSION}

\subsection{Time-dependent Heating Models}

We obtain time-dependent heating models by starting from the initial atmosphere and inputting wave energies in form of magnetic and acoustic waves. They are gradually injected into the atmospheres over a timespan of about three wave periods. This process calms down the switch-on effects, thus allowing to obtain plausible outcomes within a reasonable computational time frame. Thereafter, waves are followed in time. Due to the outward decrease in gas density and pressure, shocks form. Shock dissipation increases the local temperatures of the chromospheric layers; they also initiate velocity fields and decisively impact the local and global atmospheric thermodynamics. 
The ionization of the $\mathrm{Ca}$ to $\mathrm{Ca}$ II atoms (singly ionized $\mathrm{Ca}$ ) as well as the $\mathrm{Mg}$ to $\mathrm{Mg}$ II (singly ionized $\mathrm{Mg}$ ) atoms are local indicators of the increase in temperatures. After the transmission of (on average) 30 shocks, the atmospheres reach a state of dynamical equilibrium, largely determined by the competition between the dissipated wave energies and the radiative energy losses.

As part of our study, we consider different types of wave computations, notably acoustic waves and LTW simulations, regarding magnetic filling factors of $f_{0}=0.3 \%$ and $1.4 \%$, respectively. In monochromatic wave models, a dynamic equilibrium is obtained after the original "switch-on" phase of the atmosphere subsided. The initial wave energy flux of the LTWs is about a factor of 5.2 greater than that of the ACWs; however, owing to the shape of the flux tubes, the wave energy flux of the LTWs is subjected to a significantly larger degree of dilution. As it turns out, there is little difference in the shock strengths in those models, which is identified as $M_{\mathrm{sh}} \approx 1.9$, largely independent of the atmospheric height.

However, significantly larger shock strength are encountered in wave models based upon frequency spectra; this is a consequence of shock-shock interaction also readily identified in previous works (e.g., Cuntz 1987; Carlsson \& Stein 1995; Ulmschneider et al. 2005; Fawzy et al. 2012). This process is especially evident in LTW models - noting that in some instances (although only temporarily) shock strengths of $M_{\mathrm{sh}} \gtrsim 20$ are found. Strong shocks have a profound impact on dynamic stellar atmospheres, including high temperatures as well as strong ionization in the post-shock regions (mostly in NTDI models). Strong temperature spikes result in strong chromospheric emission, notably in the $\mathrm{Ca}$ II and $\mathrm{Mg}$ II lines. However, strong shocks also cause significant events of momentum transfer, leading to atmospheric expansion, i.e., rapid episodic outflows, associated with global cooling. Thus, in those models complex structures of the overall thermodynamics and atmospheric radiative environment occur.

\subsection{Effects of Time-dependent Hydrogen Ionization}

The importance of time-dependent ionization of hydrogen (as well as of other species) in outer stellar layers has been pointed out in many studies; see, e.g., Kneer (1980) and subsequent work. These studies indicate that the time scales of the plasma reaction to, e.g., the passage of shocks and the time scale associated with hydrogen ionization processes do not coincide. Sudden fluctuations in the plasma temperature are not mirrored by instant changes in the hydrogen ionization degree. The radiative transfer equations in combination with the statistical rate equations are solved for the NLTE populations while also taking into account pseudo-partial redistribution (pseudoPRD). The current computations are based on the approach given by Rammacher \& Ulmschneider (2003) and subsequent work.

Our results show that the time-averaged degree of hydrogen ionizations increases as a function of height. Figure 4 depicts a time series of similar wave phases at about the same time steps. Here we study LTWs (monochromatic waves) with period of $60 \mathrm{~s}$ and an initial magnetic energy of $F_{\mathrm{LTW}}=1.8 \times 10^{8} \mathrm{erg} \mathrm{cm}^{-2} \mathrm{~s}^{-1}$ combined with a magnetic filling factor of $f_{0}=0.3 \%$. We convey the $\mathrm{H}, \mathrm{Mg}$, and $\mathrm{Ca}$ ionization levels as a function of height for models with time-dependent ionization omitted (see Figs. 4a, 4b, 4c) versus time-dependent ionization included (see Figs. 4d, 4e, 4f).

Comparing Figs. $4 \mathrm{a}$ and $4 \mathrm{~d}$ reveals that the ionization levels of hydrogen are strongly correlated with the shock strength and the corresponding temperature jumps. For example, after the passage at about $2500 \mathrm{~s}$, a steady state of the hydrogen ionization is attained. However, the saturation level of the degree of hydrogen ionization does not exceed $20 \%$ contrary to the levels of $\mathrm{Mg}$ and $\mathrm{Ca}$ ionization. Regarding Figs. 4a and 4c, a clear saturation in the hydrogen ionization degree is reached around a height of $2300 \mathrm{~km}$. Some similarities in behavior are also found for models without time-dependent ionization as in those models strict correlations between, e.g., the levels of hydrogen ionization and the instant changes in the local temperatures occur (see Figs. 4b and 4c). The hydrogen ionization levels reach a steady state after around $1250 \mathrm{~s}$, with levels of about $90 \%$ above a height of $2000 \mathrm{~km}$; this value of hydrogen ionization is considerably higher than in the corresponding TDI models. In fact, models with TDI experience saturation in regard to hydrogen ionization at mostly 20\%; see comparison between Figs. 4a and $4 \mathrm{~d}$.

In summary, the time-dependent ionization of hydrogen leads to a permanent increase in $\mathrm{Mg}$ II ionization as a function of height and is uncoupled with the local temperature shocks. This behavior is not observed in NTDI models, where the degree of hydrogen ionization is largely coupled with the passage of local shocks and temperature changes.

\subsection{Properties of Time-averaged Atmospheres}

Information on the properties of time-averaged atmospheres is given in Figures 5 through 8. In the first two of these figures, we focus on the temperature, the hydrogen ionization degree, and the Ca II and $\mathrm{Mg}$ II ionization of the various models. For the sets of monochromatic waves, the averaging in time has been pursued after the "switch-on" phases have been subsided, which typically corresponds to about 30 wave periods. Regarding spectral waves, the "switch-on" phenomenon persists, as expected; however, averages have been calculated over sufficiently long time spans in order to obtain meaningful values.

The mean atmospheric temperatures are found to be highest for monochromatic waves compared to spectral waves, which is due to the fact that the very strong shocks initiated by shock-shock interaction (i.e., merging) in spectral wave models result in strong global cooling, thus counteracting the strong episodic heating by those shocks. Furthermore, at large chromospheric heights, the mean atmospheric temperatures are found to be highest in models of TDI compared to NTDI. This behavior is attributable to the differences in the shock strengths between the models.

Moreover, the degree of hydrogen ionization, on average, increases with atmospheric height in all models. This behavior is as expected, and it is a direct consequence of shock heating pertaining to atmospheres that are shaped by a decrease in density as a function of atmospheric height. However, the degrees of both $\mathrm{Ca}$ II/Ca and $\mathrm{Mg} \mathrm{II/Mg}$ notably decrease as a function of atmospheric height; this is a direct consequence of the occurrence of $\mathrm{Ca}$ III and $\mathrm{Mg}$ III due to further ionization as caused by shock heating. Clearly, the number densities of $\mathrm{Ca}$ III and $\mathrm{Mg}$ III are negligible at lower heights.

Furthermore, models of different magnetic filling factors $f_{0}$ indicate that larger filling factors correspond to higher (on average) chromospheric temperatures. Moreover, those models are also characterized by the highest $\mathrm{Ca}$ II/Ca ionization rates, especially in the upper magnetically heated chromospheres. As previously discussed in the literature see, e.g., Cuntz et al. (1999) and Fawzy et al. (2002b), magnetic wave heating readily exceeds the heating potential of acoustic waves, especially in models of high magnetic filling factors.

At large chromospheric heights, there are also notable differences in the behavior of the mechanical energy fluxes with respect to the models incorporating TDI or NTDI; see Fig. 7. TDI models heated by monochromatic or spectral waves show a more rapid dilution of the wave energy flux as a function of height compared NTDI models. 


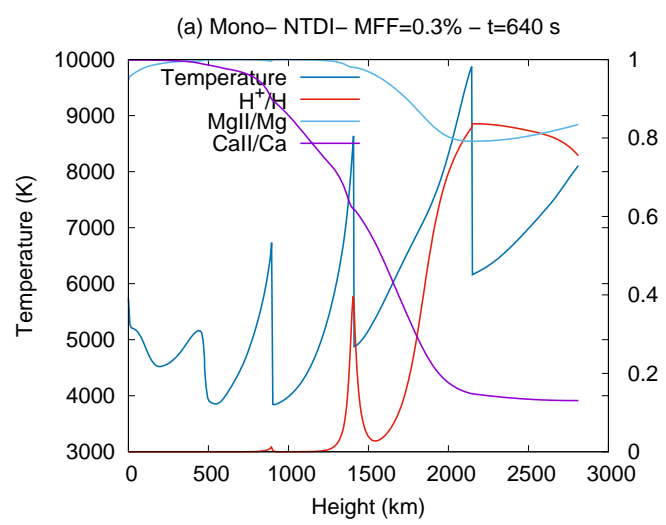

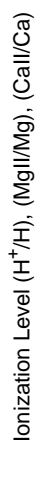

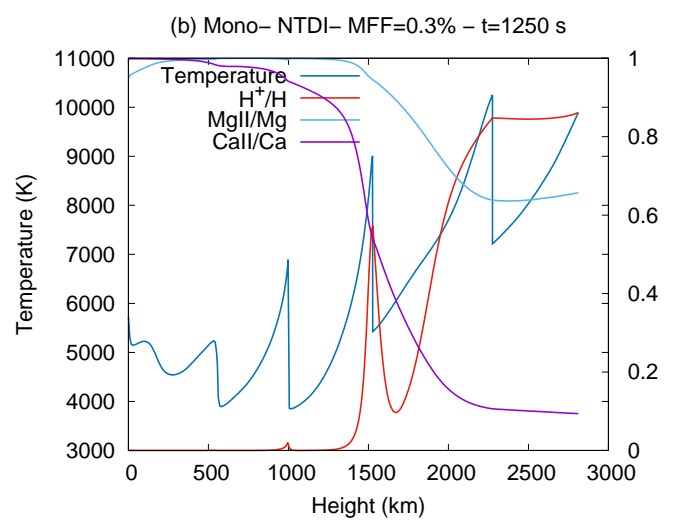

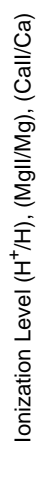

(c) Mono- NTDI- MFF=0.3\% - $\mathrm{t}=2500 \mathrm{~s}$

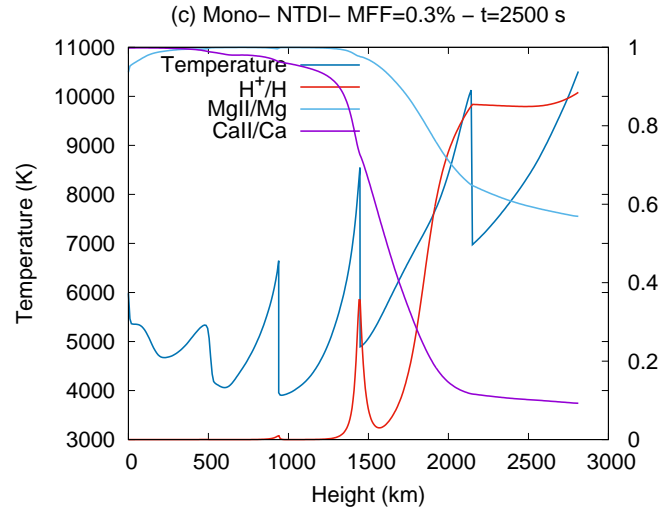

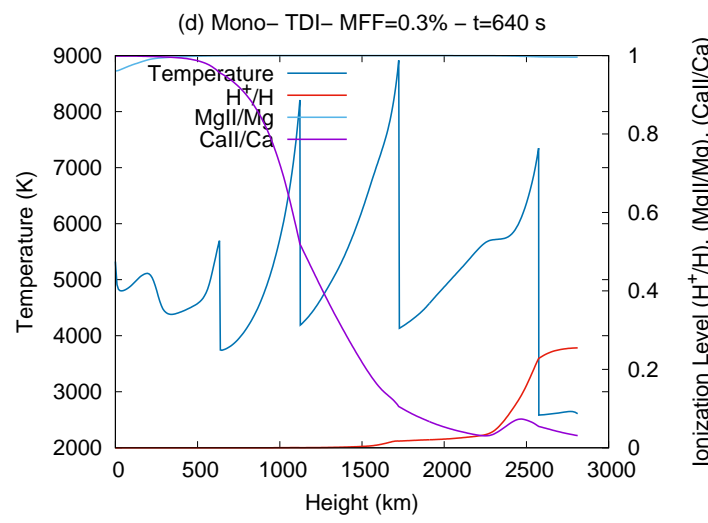
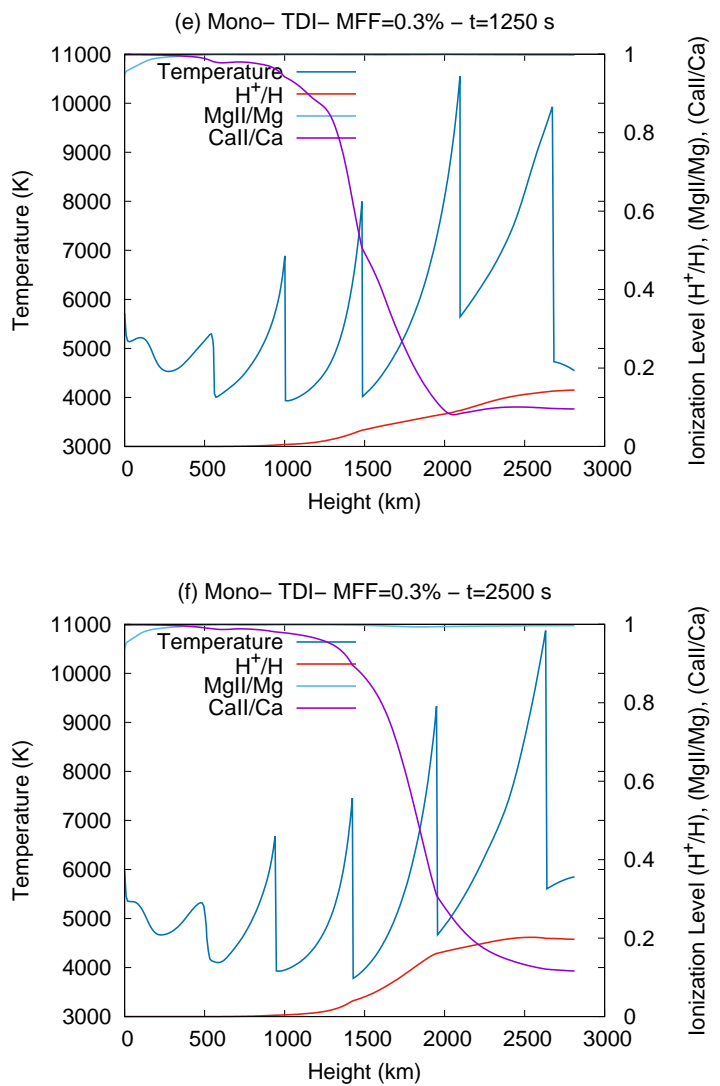

Figure 4. Snapshots of wave phases at different elapsed times for models based on time-independent hydrogen ionization (left panel) and time-dependent hydrogen ionization (right panel). We convey the temperature, and the $\mathrm{H}^{+} / \mathrm{H}, \mathrm{Ca} \mathrm{II} / \mathrm{Ca}$ and $\mathrm{Mg} \mathrm{II} / \mathrm{Mg}$ ionization levels as a function of the atmospheric height.

This behavior is due to the fact that in TDI models (especially those based on wave spectra), Ca II and Mg II are mostly ionized to Ca III and $\mathrm{Mg}$ III, respectively, at those heights.

Another interesting property is that increases in the initial mechanical energy fluxes (as explored for LTW models) are essentially inconsequential at intermediate and large chromospheric heights. This is a consequence of the well-known limiting shock-strength behavior, as established for monochromatic waves (e.g., Cuntz 2004), but in terms of the wave energy flux also encountered by spectral waves. In fact, as depicted by Table 3, only 1 to $4 \%$ of the initial total wave energy flux is converted to $\mathrm{Ca}$ II $\mathrm{K}$ emission, while the lion's share of that flux (including in models with the initial total wave energy flux increased) is converted to $\mathrm{H}^{-}$emission.
Moreover, our simulations also indicate (see Fig. 8) that the mean gas speed increases as a function of height. The highest speeds are obtained in the upper chromosphere regarding spectral wave models. This outcome is directly associated with the impact of strong shocks in those models considering that they are able to facilitate the largest amount of momentum transfer. The difference between models based on spectral and monochromatic waves is about a factor of 5 .

\subsection{Studies of Ca II H+K emission}

\subsubsection{Results for Two-Component Wave Models}

Finally, we focus on studies of Ca II emission based on our various sets of models. The computation of the emergent Ca II emission 

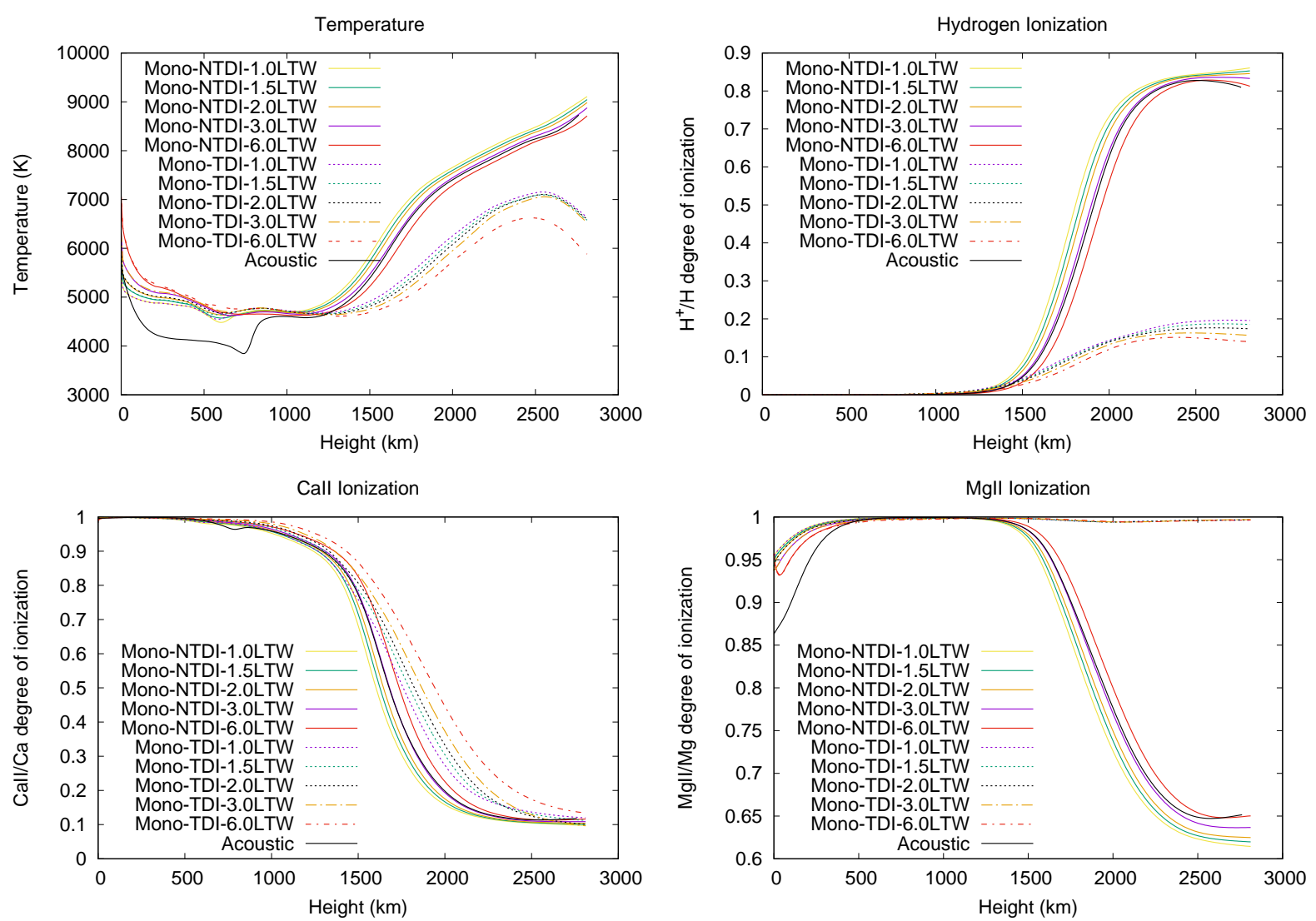

Figure 5. Results for time-averaged atmospheres for different sets of models based on NTDI and TDI simulations while assuming different wave energy fluxes and $f_{0}=0.3 \%$. All models pertain to monochromatic waves. We depict the atmospheric temperature, $\mathrm{H}^{+} / \mathrm{H}$ ionization degree, the Ca II/Ca ionization degree, and the $\mathrm{Mg} \mathrm{II} / \mathrm{Mg}$ ionization degree. For comparison we also give the results for acoustic waves (monochromatic, TDI).

fluxes for the different types of theoretical models follows the approach outlined in Sect. 2.4 and 2.5. It ensures that all surface areas contribute to the computations of the disk-integrated Ca II emission, which is done through consideration of different angles of view.

Table 3 conveys the Ca II K surface integrated fluxes of the various models. Regarding TDI models with initial wave energy fluxes of 1.0 $\times$ LTW and $f_{0}=0.3 \%$, it is found that the emergent Ca II K for the core emission (defined over a wavelength range of $\Delta \lambda=$ \pm 0.3 ) is approximately $6 \%$ higher for spectral waves compared to monochromatic waves - which is due to strong shocks occurring in spectral wave models due to shock-shock interaction, which is largely absent in monochromatic models. Table 4 shows that this trend also applies to models with $f_{0}=1.4 \%$. Here the emergent $\mathrm{Ca}$ II $\mathrm{K}$ for the core emission is about $15 \%$ higher for spectral waves compared to monochromatic waves. The corresponding Ca II K output for acoustic waves (spectral models) is reduced by $38 \%$ and $65 \%$, respectively, relative to the LTW models with $f_{0}=0.3 \%$ and $1.4 \%$. Models employing the NTDI assumption tend to show higher values for the emergent $\mathrm{Ca}$ II K core emission as in those models — at least occasionally — higher radiative emissions in the post-shock regions occur.

In our reference models, the computation of the emergent Ca II fluxes are based on instantaneous time-sequences of about 10 wave phases with shocks, assessed over timespans of approximately $55 \mathrm{~s}$. In order to study the relevance of nonlinearities, as imposed by the shocks, including the associated effects on the emergent $\mathrm{Ca}$ II fluxes, we also generated time-averages for every five phases over timespans of about $27.5 \mathrm{~s}$. The comparisons to the instantaneous models reveal that the total emergent Ca II fluxes (line cores and wings) are about the same for both cases, but the time-averaged core flux is reduced by about $20 \%$ compared to the value obtained by the instantaneous reference model. Figure 9 shows the results for an LTW model heated by monochromatic waves with time-dependent ionization and a magnetic filling factor $f_{0}=0.3 \%$.

\subsubsection{Consideration of Mode Coupling}

Our main models consider two-component chromospheres based on ACWs and LTWs. However, we augmented this approach through the consideration of torsional magnetic tube waves (TTWs); e.g., Noble et al. (2003) for a detailed analysis of TTW wave energy generation with applications to the Sun. TTWs are readily excited in flux tubes through foot-point motions. In the context of this work, adopted wave energy fluxes of TTWs are expressed in multiples of the wave energy fluxes of LTWs. This approach is also used in assessments of Ca II emission.

The power spectrum analysis of the vertical and horizontal velocity fluctuations in the Imaging Magnetograph eXperiment (IMaX) data revealed the existence of the interactions between longitudinal and transverse wave oscillations in small scale magnetic features (Stangalini et al. 2013). Hasan et al. (2003) previously showed that the nonlinear coupling of LTWs and TTWs plays an important role in the heating of upper solar chromospheric layers. The heights of shock formation for both types of waves have been identified as 

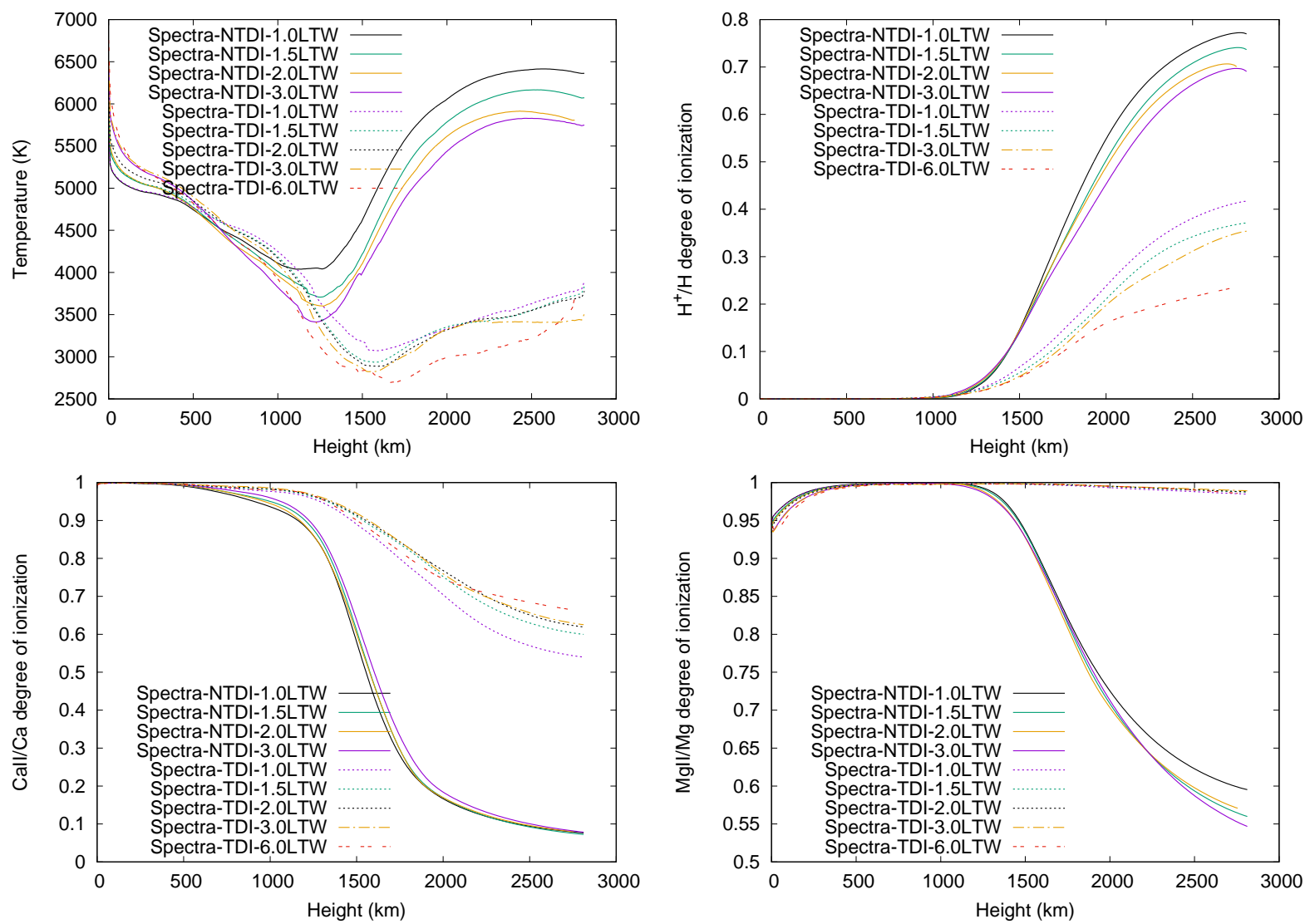

Figure 6. Same as Fig. 5, but pertaining to spectral wave models.

relatively similar, and after shocks are formed the mode coupling remains highly efficient, thus effectively contributing to chromospheric heating and emission.

In the view of these findings, we also computed models while employing linear increases in the input mechanical energy for LTWs with factors ranging from 0.5 to 6 times of the initial mechanical energy carried by LTWs; see Table 3 for information. It was found that the obtained emitted Ca II fluxes are strongly correlated with the amount of input mechanical energy up to a value of about $3.5 \times$ LTW; however, the emitted fluxes reached distinct levels of saturation (depending on whether the TDI or NTDI assumption is employed). This can be explained by the fact that the heating by wave coupling reaches a limit, implying that other mechanisms would be required to increase the amount of wave energies any further. In case of strong shocks, associated with higher wave energy fluxes, high amounts of momentum transfer occur entailing global cooling, which tends to counteract the heating initiated by the strong shocks. Results for the Ca II K core fluxes (defined over a wavelength range of $\Delta \lambda= \pm 0.3$ ) for different types of models are displayed in Figure 10. Here $\Delta \lambda$ is calculated relative to the unheated atmosphere; thus, the subtraction of the photospheric contribution is readily considered.

\section{SUMMARY AND CONCLUSIONS}

We pursued various chromospheric heating calculations for $55 \mathrm{Cnc}$, a main-sequence star of spectral-type late-G; this star generally exhibits a low level of chromospheric activity. This behavior stems from its old age (Mamajek \& Hillenbrand 2008; von Braun et al. 2011;
Yee et al. 2017) and slow rotation (Henry et al. 2000; Bourrier et al. 2018). Previously developed codes allow us addressing the different steps of the magneto-acoustic heating picture ${ }^{3}$, comprised of convective energy generation (e.g., Musielak et al. 1994, 1995; Ulmschneider et al. 2001), the propagation and dissipation of waves through different layers of the stellar atmosphere (e.g., Buchholz et al. 1998; Fawzy et al. 2002a), and the emergence of radiative energy output (e.g., Cuntz et al. 1999; Fawzy et al. 2002a).

Besides acoustic waves, our models prominently also take into account longitudinal flux tube waves to address magnetic heating (e.g., Narain \& Ulmschneider 1996). LTWs are constraint by 55 Cnc's stellar parameters, notably its rotation period, which in the framework of our model allows us to describe the photospheric and chromospheric magnetic filling factors; see, e.g., Cuntz et al. (1999) for a discussion of the inherent features and limitations of this approach. Previous models for $\epsilon$ Eri based on this kind of approach were given by Fawzy \& Cuntz (2018). Moreover, we calculated additional models for $55 \mathrm{Cnc}$ that consider the time-dependent energy deposition by transverse tube waves in an approximate manner.

The emergent Ca II K fluxes, an important component of the overall chromospheric energy losses, have been computed in detail (multiray treatment) assuming partial redistribution in combination with time-dependent hydrogen ionization (main models). Here as well as in our previous papers, these fluxes have been calculated and interpreted for different levels of stellar magnetic activity, representative

3 We contend that there is a large body of literature on that topic. The following references are given as examples only; however, they are most closely associated with the code package utilized by the authors. 

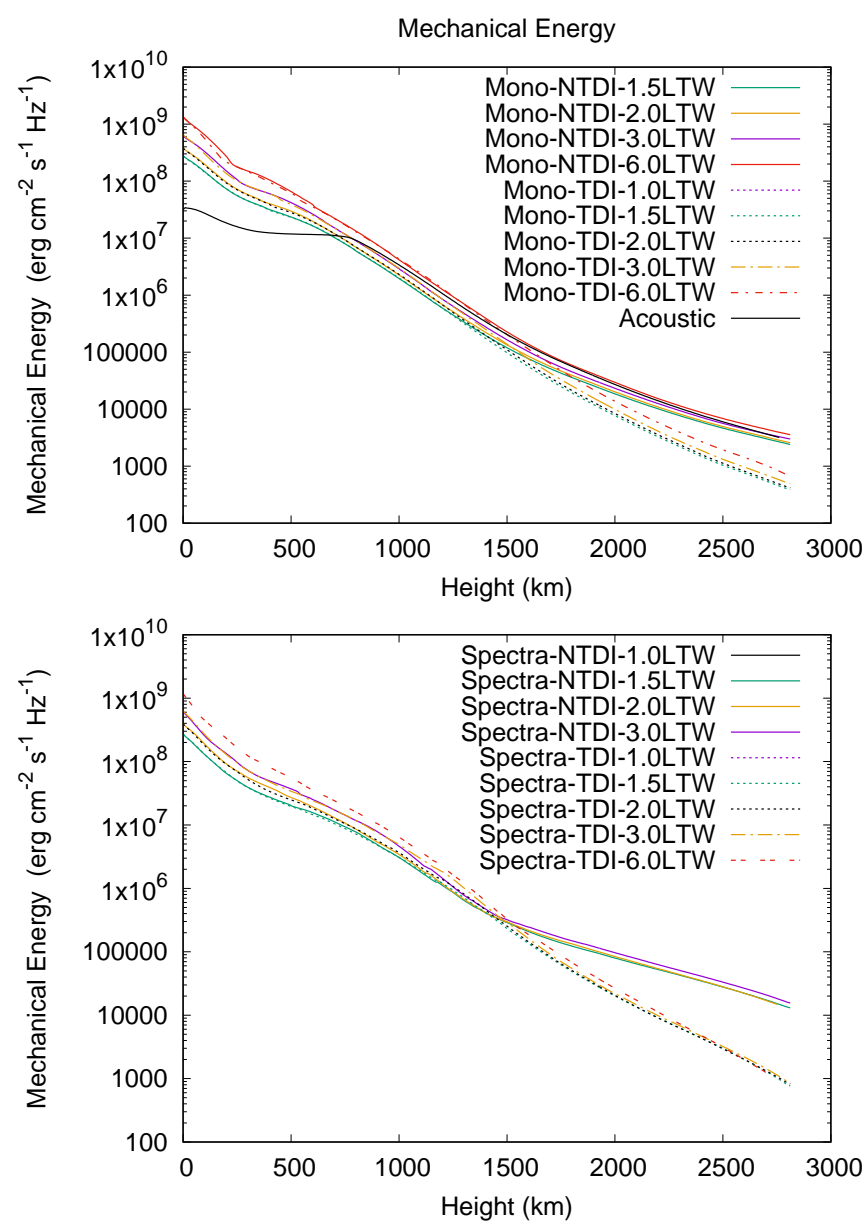

Figure 7. Behavior of the mechanical energy flux as a function of height for different sets of models. The top panel conveys monochromatic wave models, whereas bottom panel conveys spectral wave models. Here we show both NTDI and TDI models while also assuming different wave energy fluxes and $f_{0}=0.3 \%$. For comparison we also give the results for acoustic waves (monochromatic, TDI).

Table 3. Ca II K SURFACE INTEGRATED FLUXES

\begin{tabular}{llcccc}
\hline \multirow{2}{*}{ Input Energy } & Item & Mono-NTDI & Mono-TDI & Spectra-NTDI & \multirow{2}{*}{ Spectra-TDI } \\
\hline $1.0 \mathrm{LTW}$ & Total Flux & $1.43 \mathrm{E}+7$ & $1.40 \mathrm{E}+7$ & $1.61 \mathrm{E}+7$ & $1.62 \mathrm{E}+7$ \\
$\ldots$ & Core Flux & $5.17 \mathrm{E}+5$ & $3.51 \mathrm{E}+5$ & $4.68 \mathrm{E}+5$ & $3.73 \mathrm{E}+5$ \\
$1.5 \mathrm{LTW}$ & Total Flux & $1.52 \mathrm{E}+7$ & $1.41 \mathrm{E}+7$ & $9.33 \mathrm{E}+6$ & $2.11 \mathrm{E}+7$ \\
$\ldots$ & Core Flux & $5.49 \mathrm{E}+5$ & $4.02 \mathrm{E}+5$ & $4.14 \mathrm{E}+5$ & $4.60 \mathrm{E}+5$ \\
$2.0 \mathrm{LTW}$ & Total Flux & $1.75 \mathrm{E}+7$ & $1.46 \mathrm{E}+7$ & $3.30 \mathrm{E}+7$ & $2.35 \mathrm{E}+7$ \\
$\ldots$ & Core Flux & $5.96 \mathrm{E}+5$ & $4.38 \mathrm{E}+5$ & $4.31 \mathrm{E}+5$ & $4.68 \mathrm{E}+5$ \\
$3.0 \mathrm{LTW}$ & Total Flux & $2.82 \mathrm{E}+7$ & $1.95 \mathrm{E}+7$ & $3.32 \mathrm{E}+7$ & $3.64 \mathrm{E}+7$ \\
$\ldots$ & Core Flux & $6.71 \mathrm{E}+5$ & $4.86 \mathrm{E}+5$ & $5.27 \mathrm{E}+5$ & $4.90 \mathrm{E}+5$ \\
$6.0 \mathrm{LTW}$ & Total Flux & $6.07 \mathrm{E}+7$ & $5.61 \mathrm{E}+7$ & $\ldots$ & $5.61 \mathrm{E}+7$ \\
$\ldots$ & Core Flux & $6.73 \mathrm{E}+5$ & $5.13 \mathrm{E}+5$ & $\ldots$ & $5.13 \mathrm{E}+5$ \\
\hline
\end{tabular}

of increased magnetic heating and emission (e.g., Cuntz et al. 1999; Fawzy et al. 2002a, 2012, and references therein). Nevertheless, our main focus relates to the low-activity stage of that star, represented by a magnetic filling factor of $f_{0}=0.3 \%$; however, additional models with $f_{0}=1.4 \%$ have been calculated as well.

We note that the collisional excitation of the Ca II upper levels of the $\mathrm{H}$ and $\mathrm{K}$ lines is proportional to the local electron density (as well as the local temperature); see, e.g., Vernazza et al. (1981).
In the chromosphere, $\mathrm{H}$ ionization is the major source of electrons; however, the ionization of $\mathrm{H}$ is very different for the NTDI and TDI models and the temperatures are very different as well. On the other hand, the Ca II emission is not very different for the NTDI and TDI models considering that the $\mathrm{Ca} \mathrm{II} \mathrm{H}+\mathrm{K}$ lines are formed over a wide range of heights and thus temperatures. Nevertheless, the electron densities notably differ between those types of models, especially behind the shocks. 

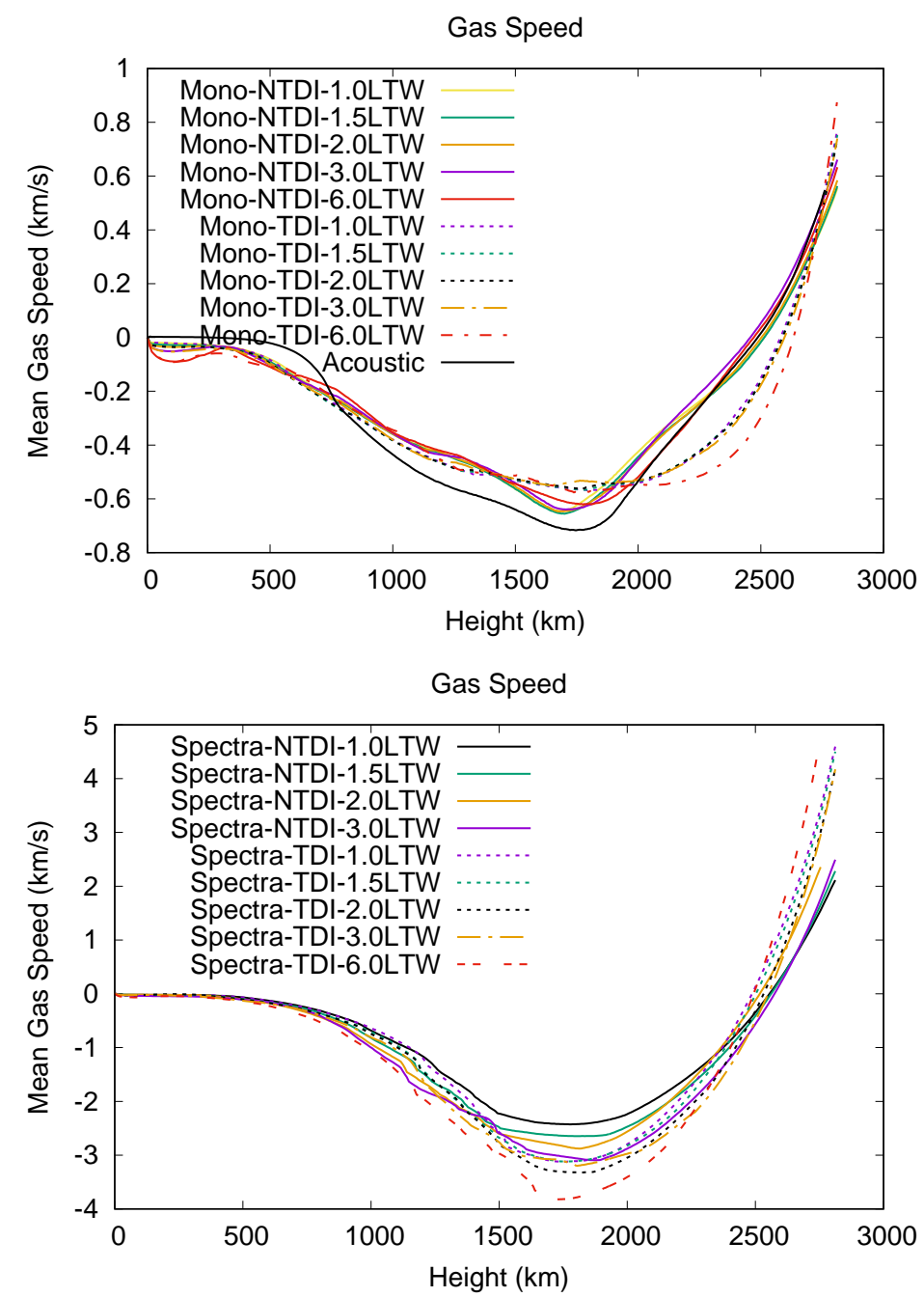

Figure 8. Behavior of the mean gas speed as a function of height for different sets of models. The top panel conveys monochromatic wave models, whereas bottom panel conveys spectral wave models. Here we show both NTDI and TDI models while assuming different wave energy fluxes and $f_{0}=0.3 \%$. For comparison we also give the results for acoustic waves (monochromatic, TDI). Note the different $y$-scales between the two panels.

Table 4. Ca II K SURFACE CORE INTEGRATED FLUXES

\begin{tabular}{cccccc}
\hline MFF & Model & Mono-NTDI & Mono-TDI & Spectra-NTDI & Spectra-TDI \\
\hline 0.0 & ACW & $4.45 \mathrm{E}+5$ & $2.87 \mathrm{E}+5$ & $4.19 \mathrm{E}+5$ & $2.30 \mathrm{E}+5$ \\
0.3 & LTW & $5.17 \mathrm{E}+5$ & $3.51 \mathrm{E}+5$ & $4.68 \mathrm{E}+5$ & $3.73 \mathrm{E}+5$ \\
1.4 & LTW & $7.13 \mathrm{E}+5$ & $5.69 \mathrm{E}+5$ & $5.73 \mathrm{E}+5$ & $6.54 \mathrm{E}+5$ \\
\hline
\end{tabular}

Based on our models, we obtained the following results:

(1) Both ACWs and LTWs form shocks in the upper photosphere / lower chromosphere In case of monochromatic waves, the height of shock formation for LTWs (with $f_{0}=0.3 \%$ ) is about $30 \mathrm{~km}$ lower in response to the higher wave energy flux. Generally, higher shock strengths are attained for spectral waves compared to monochromatic waves. This result agrees with previous findings (e.g., Cuntz et al. 1998, 1999; Fawzy \& Cuntz 2018). Notable differences between the models occur at larger heights, mostly due the impact of dilution of the wave energy flux.
(2) The mean atmospheric temperatures are highest in monochromatic wave models compared to spectral wave models. Regarding both monochromatic and spectral models, NTDI models exhibit a spatial increase in temperature contrary to TDI models. This behavior is attributable to the differences in the shock strengths between those models.

(3) The formation heights for Ca II and Mg II range between for $700 \mathrm{~km}$ and $1800 \mathrm{~km}$, somewhat depending on the model. Radiative energy losses are most pronounced behind strong shocks owing to the impact of shock-shock interaction and in models with time-dependent hydrogen ionization omitted. Peaks of $\mathrm{Ca}$ II and $\mathrm{Mg}$ II emission 


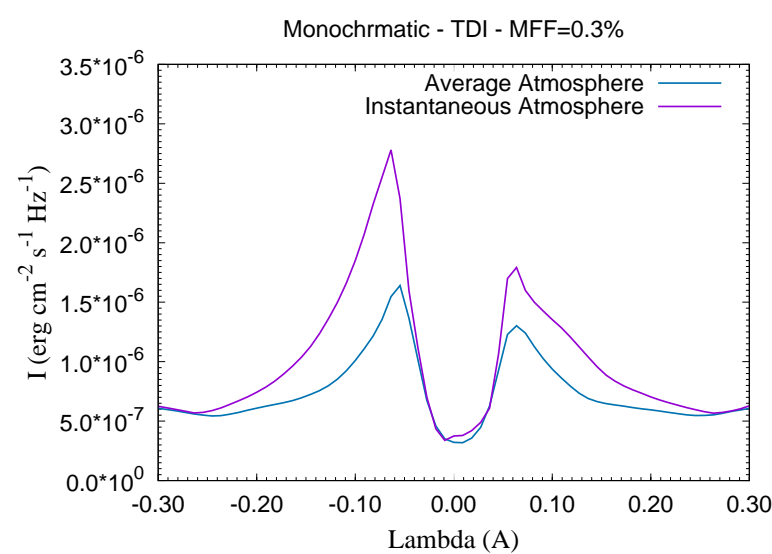

Figure 9. Ca II K profiles for an LTW TDI model heated by monochromatic waves based on the instantaneous and the time-averaged atmosphere.

behind shocks (including those of large strengths) do not occur in TDI models owing to the difference in the time scales between the ionization processes and shock propagation.

(4) Models of different magnetic filling factors $f_{0}$ indicate that a larger filling factor corresponds to a higher (on average) chromospheric temperatures. Moreover, those models are also characterized by the highest $\mathrm{Ca} \mathrm{II} / \mathrm{Ca}$ ionization rates, especially in the upper magnetically heated chromospheres. This result is relevant for stages of enhanced stellar magnetic activity; see, e.g., Fawzy et al. (2002b) for previous results - as well as for stars other than $55 \mathrm{Cnc}$.

(5) Increased initial mechanical energy fluxes (as explored for LTW models) are essentially inconsequential at intermediate and large chromospheric heights for the atmospheric radiative energy losses, a consequence of the limiting shock-strength behavior, as established and partially also encountered by spectral waves. In fact, only 1 to $4 \%$ of the initial total wave energy flux is converted to Ca II K emission, while most of that flux (including in models with the initial total wave energy flux increased) is converted to $\mathrm{H}^{-}$emission.

(6) At large chromospheric heights, notable differences exist in the behavior of the mechanical energy flux for models based on TDI versus those based on the NTDI approximation. TDI models heated by monochromatic or spectral waves show a faster dilution of the wave energy flux at larger heights compared to NTDI models. This is because Ca II and $\mathrm{Mg}$ II are mostly ionized to Ca III and Mg III, respectively, at those heights, especially in TDI models employing wave spectra.

(7) Regarding the averaged Ca II core fluxes, we also investigated the impact of increased initial wave energy fluxes. We found that the emitted Ca II fluxes are strongly correlated with the amount of the inputted wave energy up to a value of about $3.5 \times$ LTW; however, thereafter a saturation level is reached. The reason is that heating by wave coupling reached its limit (in part caused by strong cooling associated with the momentum transfer due to strong shocks). Hence, other mechanisms would be required to further increase the overall atmospheric thermal energy budget.

(8) Our study on $55 \mathrm{Cnc}$, based on the propagation and dissipation of waves, constitutes another opportunity to illustrate the significance of nonlinearities. Assuming LTW TDI models with $f_{0}=0.3 \%$ heated by monochromatic waves based on either the instantaneous or the time-averaged atmosphere indicates that the $\mathrm{Ca}$ II $\mathrm{K}$ core flux is significantly reduced if based on a time-averaged model atmosphere relative to the (realistic) instantaneous atmosphere.
The aim of this study was a detailed assessment of LTW and ACW models for $55 \mathrm{Cnc}$ pertaining to magnetic and nonmagnetic regions, respectively. The next article of this series (Paper II) will feature a comparison between our theoretical models and observations.

\section{ACKNOWLEDGEMENTS}

This research is supported in part by the Izmir University of Economics (D. E. F.) and the University of Texas at Arlington (M. C). Furthermore, we are grateful to M. Sosebee (UTA, Dep. of Physics) for his assistance with computer graphics.

\section{DATA AVAILABILITY}

The data underlying this article will be shared on reasonable request to the corresponding author.

\section{REFERENCES}

Bourrier V., et al., 2018, A\&A, 619, A1

Buchholz B., Ulmschneider P., Cuntz M., 1998, ApJ, 494, 700

Carlsson M., Stein R. F., 1995, ApJ, 440, L29

Charbonneau P., Schrijver C. J., MacGregor K. B., 1997, in Cosmic Winds and the Heliosphere, eds. J. R. Jokipii et al. (Tucson: Univ. of Arizona Press), 677

Cuntz M., 1987, A\&A, 188, L5

Cuntz M., 2004, A\&A, 420, 699

Cuntz M., Ulmschneider P., Musielak Z. E., 1998, ApJ, 493, L117

Cuntz M., Rammacher W., Ulmschneider P., Musielak Z. E., Saar S. H., 1999, ApJ, 522, 1053

Fawzy D. E., 2015, Ap\&SS, 357, 125

Fawzy D. E., Cuntz M., 2011, A\&A, 526, A91

Fawzy D. E., Cuntz M., 2018, Ap\&SS, 363, 152

Fawzy D. E., Ulmschneider P., Cuntz M., 1998, A\&A, 336, 1029

Fawzy D., Rammacher W., Ulmschneider P., Musielak Z. E., Stępień K., 2002a, A\&A, 386, 971

Fawzy D., Stẹpień K., Ulmschneider P., Rammacher W., Musielak Z. E., 2002b, A\&A, 386, 994

Fawzy D. E., Cuntz M., Rammacher W., 2012, MNRAS, 426, 1916

Freij N., Scullion E. M., Nelson C. J., Mumford S., Wedemeyer S., Erdélyi R., 2014, ApJ, 791, 61

Gonzalez G., 1998, A\&A, 334, 221

Gray D. F., 2005, The Observation and Analysis of Stellar Photospheres (3rd edn., Cambridge, UK: Cambridge Univ. Press)

Gray R. O., Corbally C. J., Garrison R. F., McFadden M. T., Robinson, P. E., 2003, AJ, 126, 2048

Hartmann L. W., Noyes R. W., 1987, ARA\&A, 25, 271

Hasan S. S., Kalkofen W., van Ballegooijen A. A., Ulmschneider P., 2003, ApJ, 585, 1138

Henry G. W., Marcy G. W., Butler R. P., Vogt S. S., 2000, ApJ, 531, 415

Herbold G., Ulmschneider P., Spruit H. C., Rosner R., 1985, A\&A, 145, 157

Hoffleit D., Warren Jr. W. H. 1995, VizieR Online Data Catalog

Johns-Krull C. M., Valenti J. A., 1996, ApJ, 459, L95

Johns-Krull C. M., Valenti J. A., 2000, in Stellar Clusters and Associations: Convection, Rotation, and Dynamos, eds. R. Pallavicin et al. (San Francisco: ASP), Astr. Soc. Pac. Conf. Ser., 198, 371

Jordan C., 1997, Astron. Geophys., 38, 10

Judge P. G., Carpenter K. G., 1998, ApJ, 494, 828

Judge P. G., Cuntz M., 1993, ApJ, 409, 776

Kayshap P., Murawski K., Srivastava A. K., Musielak Z. E., Dwivedi B. N., 2018, MNRAS, 479, 5512

Keppens R., MacGregor K. B., Charbonneau P., 1995, A\&A, 294, 469

Kneer F., 1980, A\&A, 87, 229

Ligi R., et al., 2016, A\&A, 586, A94 


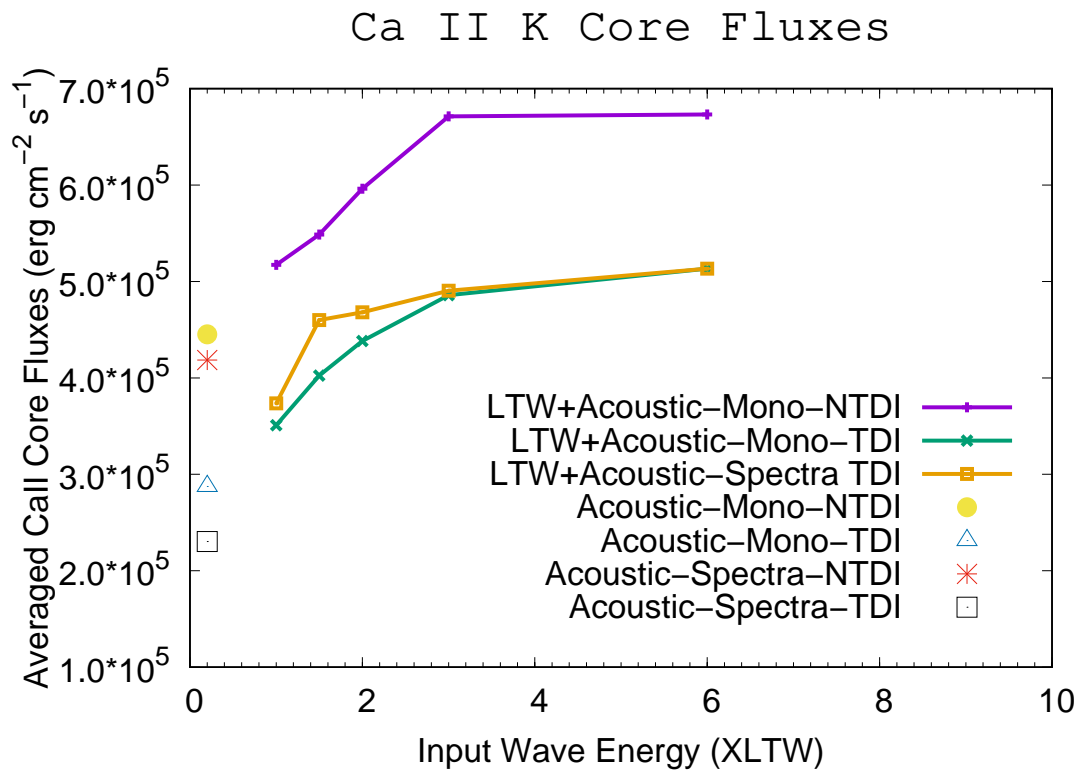

Figure 10. Averaged Ca II K core fluxes for different types of models as a function of multiples of LTW wave energies based on $f_{0}=0.3 \%$. Results are given for monochromatic NTDI and TDI models, as well as spectral TDI models. Moreover, we also give the results for the various models based on acoustic waves.

Linsky J. L., 1983, in Solar and Stellar Magnetic Fields: Origins and Coronal Effects, ed. J. Stenflo (Dordrecht: Reidel), 313

Mamajek E. E., Hillenbrand L. A., 2008, ApJ, 687, 1264

Marcy G. W., Basri G., 1989, ApJ, 345, 480

Marilli E., Catalano S., Trigilio C., 1986, A\&A, 167, 297

Mittag M., Schmitt J. H. M. M., Schröder K.-P., 2013, A\&A, 549, A117

Mittag M., Schmitt J. H. M. M., Schröder K.-P., 2018, A\&A, 618, A48

Montesinos B., Jordan C., 1993, MNRAS, 264, 900

Musielak Z. E., Rosner R., Stein R. F., Ulmschneider P., 1994, ApJ, 423, 474

Musielak Z. E., Rosner R., Gail H. P., Ulmschneider P., 1995, ApJ, 448, 865

Narain U., Ulmschneider P., 1996, Space Sci. Rev., 75, 453

Noble M. W., Musielak Z. E., Ulmschneider P., 2003, A\&A, 409, 1085

Noyes R. W., Hartmann L. W., Baliunas S. L., Duncan D. K., Vaughan A. H., 1984, ApJ, 279, 763

Rammacher W., Cuntz M., 2003, ApJ, 594, L51

Rammacher W., Ulmschneider P., 2003, ApJ, 589, 988

Rüedi I., Solanki S. K., Mathys G., Saar S. H., 1997, A\&A, 318, 429

Saar S. H., 1996, in Stellar Surface Structure, IAU Symp. 176, ed. K. G. Strassmeier \& J. L. Linsky (Dordrecht: Kluwer), 237

Schrijver C. J., 1995, A\&ARv, 6, 181

Schrijver C. J., Zwaan C. 2000, Solar and Stellar Magnetic Activity (Cambridge, UK: Cambridge Univ. Press)

Schrijver C. J., Coté J., Zwaan C., Saar S. H., 1989, ApJ, 337, 964

See V., et al., 2019, ApJ, 876, 118

Skumanich A., 1972, ApJ, 171, 565

Stangalini M., Solanki S. K., Cameron R., Martínez Pillet V., 2013, A\&A, 554, A115

Stein R. F., Nordlund Å., Georgobiani D., Benson D., Schaffenberger W., 2009a, in Solar-Stellar Dynamos as Revealed by Helio- and Asteroseismology, eds. M. Dikpati et al. (San Francisco: ASP), Astr. Soc. Pac. Conf. Ser., 416, 421

Stein R. F., Georgobiani D., Schaffenberger W., Nordlund Å., Benson D., 2009b, in Cool Stars, Stellar Systems, and the Sun 15, ed. E. Stempels (New York: AIP), Am. Inst. Phys., 1094, 764

Spruit H. C., 1981, A\&A, 102, 129

Ulmschneider P., Musielak Z. E., 1998 A\&A, 338, 311

Ulmschneider P., Theurer J., Musielak Z. E., 1996 A\&A, 315, 212

Ulmschneider P., Musielak Z. E., Fawzy D. E., 2001, A\&A, 374, 662

Ulmschneider P., Rammacher W., Musielak Z. E., Kalkofen W., 2005, ApJ, 631, L155
Vernazza J. E., Avrett E. H., Loeser R., 1981, ApJS, 45, 635

von Braun K., et al., 2011, ApJ, 740, 49

Wolff S. C., Simon T., 1997, PASP, 109, 759

Yee S. W., et al., 2017, ApJ, 836, 77

This paper has been typeset from a $\mathrm{T}_{\mathrm{E}} \mathrm{X} / \mathrm{L} \mathrm{T}_{\mathrm{E}} \mathrm{X}$ file prepared by the author. 